\title{
Risk Prediction Model of Gestational Diabetes Mellitus in a Chinese Population Based on a Risk Scoring System
}

\author{
Yanmei Wang · Zhijuan Ge $\cdot$ Lei Chen · Jun Hu • Wenting Zhou • \\ Shanmei Shen · Dalong Zhu $\cdot$ Yan Bi
}

Received: March 14, 2021 / Accepted: April 21, 2021 / Published online: May 15, 2021

(C) The Author(s) 2021

\begin{abstract}
Introduction: Gestational diabetes mellitus (GDM) is associated with adverse perinatal outcomes. Accurate models for early prediction of GDM are lacking. This study aimed to explore an early risk prediction model to identify
\end{abstract}

Yanmei Wang, Zhijuan Ge and Lei Chen equally contributed to the manuscript.

Yan Bi and Dalong Zhu are co-corresponding authors.

Supplementary Information The online version contains supplementary material available at https:// doi.org/10.1007/s13300-021-01066-2.

Y. Wang · Z. Ge · J. Hu · S. Shen · D. Zhu (凶) .

Y. Bi (ه)

Department of Endocrinology, Nanjing Drum

Tower Hospital, The Affiliated Hospital of Nanjing

University Medical School, No. 321, Zhongshan

Road, Nanjing 210008, China

e-mail: zhudalong@nju.edu.cn

Y. Bi

e-mail: biyan@nju.edu.cn

L. Chen

Department of Endocrinology, Suzhou Hospital Affiliated to Nanjing Medical University, Suzhou, China

\section{W. Zhou}

Department of Endocrinology, Medical School of Southeast University Nanjing Drum Tower Hospital, Nanjing, China women at high risk of GDM through a risk scoring system.

Methods: This was a retrospective cohort study of 785 control pregnancies and 855 women with GDM. Maternal clinical characteristics and biochemical measures were extracted from the medical records. Logistic regression analysis was used to obtain coefficients of selected predictors for GDM in the training cohort. The discrimination and calibration of the risk scores were evaluated by the receiver-operating characteristic (ROC) curve and a Hosmer-Lemeshow test in the internal and external validation cohort, respectively.

Results: In the training cohort (total $=1640$ ), two risk scores were developed, one including predictors collected at the first antenatal care visit for early prediction of GDM, such as age, height, pre-pregnancy body mass index, educational background, family history of diabetes, menstrual history, history of cesarean delivery, GDM, polycystic ovary syndrome, hypertension, and fasting blood glucose (FBG), and the total risk score also including FBG and triglyceride values during 14-20 gestational weeks. Our total risk score yielded an area under the curve (AUC) of 0.845 (95\% CI $=0.805-0.884)$. This performed better in an external validation cohort, with an AUC of 0.886 (95\% CI $=0.856-0.916)$.

Conclusion: The GDM risk score, which incorporates several potential clinical features with routine biochemical measures of GDM, appears to be a sensitive and reliable screening tool for earlier detection of GDM risk. 
Keywords: Early pregnancy; Gestational diabetes; Prediction model; Risk score

\section{Key Summary Points}

Why carry out this study?

Gestational diabetes mellitus (GDM) is prevalent worldwide, and the epidemic of GDM is a great threat to maternal and neonatal health.

Accurate models for GDM prediction in pregnancies before the diagnosis of GDM between 24 and 28 weeks of gestation are lacking.

The aim of this study is to develop a risk scoring system to identify women at high risk of GDM in a Chinese population.

\section{What was learned from the study?}

The risk scoring system derived from clinical and biochemical predictors presented a good ability to discriminate pregnancies with increased risk of GDM. It performed well in both the internal and the external validation cohorts, with an area under the receiver-operating characteristic curve (AUC) of 0.845 (95\% $\mathrm{CI}=0.805-0.884)$ and $0.886(95 \%$ $\mathrm{CI}=0.856-0.916)$, respectively.

\section{DIGITAL FEATURES}

This article is published with digital features, including a summary slide, to facilitate understanding of the article. To view digital features for this article go to https://doi.org/10.6084/ m9.figshare.14454351.

\section{INTRODUCTION}

Gestational diabetes mellitus (GDM) is prevalent worldwide, with prevalence rates continuing to rise internationally, resulting in a significant threat to health resources $[1,2]$. GDM is associated with adverse pregnancy outcomes such as preeclampsia, shoulder dystocia, birth injury, and abnormal infant growth $[3,4]$. Moreover, GDM is known to increase the risk of developing postpartum diabetes mellitus and long-term metabolic disorders in the offspring during teenage years [5-8]. There is growing interest in our ability to identify which women will develop GDM, given that there is now emerging evidence that GDM may be preventable by interventions applied in early pregnancy $[9,10]$. Thus, it is essential for health care providers to establish an early, accurate, and affordable screening tool to assess GDM risk, so that vulnerable women may receive targeted early prevention and intervention.

Currently, clinical guidelines and consensuses suggest testing for GDM at 24-28 weeks of gestation in pregnant women not previously found to have diabetes $[11,12]$. For pregnant women with high-risk factors for diabetes mellitus, prioritizing the target group for an early diagnostic oral glucose tolerance test (OGTT) is recommended at the first prenatal visit $[11,12]$. However, the accuracy of this assessment for identifying women at high risk of GDM in early pregnancy is unclear. Furthermore, there is no international consensus for a preferred screening method or detection strategy. The current guidelines only suggest selective testing based on a maternal clinical history of risk factors, yet, this binary approach is limited by poor sensitivity and specificity.

Two recent studies have shown that clinical multivariate GDM risk prediction models can accurately identify GDM in early pregnancy, with an area under the curves (AUC) of 0.752 and 0.88 , respectively [13, 14]. Furthermore, later studies showed that risk prediction models that considered specific biomarkers such as pregnancy-associated plasma protein A (PAPPA), tissue plasminogen activator (t-PA), triglycerides (TG), or lipocalin-2 may potentially improve the early pregnancy prediction of GDM with AUCs between 0.861 and 0.96 [15-17]. However, these novel biochemical measures have not been thoroughly examined and the equations are complex, which makes these 
prediction models difficult to use in clinical applications.

Based on these findings, we sought to develop a simple risk score system for GDM prediction that could be further improved by routinely incorporating maternal demographic and clinical variables and, importantly, a range of biochemical tests that are commonly available in most laboratories.

\section{METHODS}

\section{Study Design}

This retrospective cohort study was performed at the Nanjing Drum Tower Hospital, The Affiliated Hospital of Nanjing University Medical School, Nanjing, China. We retrospectively analyzed the electronic records of women with singleton pregnancies that displayed normal glucose tolerance (NGT) or GDM who had delivered infants at our hospital. A total of 1640 pregnant women were included for retrospective analysis. According to the ratio of 7:3, 1150 pregnant women who visited our hospital from January 1, 2015, to May 21, 2017, were selected as the training cohort, while 490 pregnant women who visited from May 22, 2017, to June 30,2018 , were selected as the internal validation cohort. As for the external validation cohort, all women delivering a live-born infant at the Affiliated Suzhou Hospital of Nanjing Medical University, Suzhou Municipal Hospital, from January 2010 to August 2020 were eligible for inclusion based on the inclusion and exclusion criteria. A total of 617 pregnant women were included in external validation analyses (Supplementary Material).

This study was approved by the Institutional Ethics Committee of Nanjing Drum Tower Hospital, The Affiliated Hospital of Nanjing University Medical School, Nanjing, China. Our study conforms to the guidelines of the Declaration of Helsinki and its later amendments. Informed consent was obtained from all patients for being included in the study. The date of approval was 29 March 2016, reference number AF/SC-08/02.0.

\section{Participants}

All enrolled patients met the following inclusion criteria: (1) GDM pregnancies were identified based on the 2010 International Association of Diabetes and Pregnancy Study Groups' criteria (IADPSG), which is assessed via a 75-g OGTT fasting blood glucose (FBG) $\geq 5.1 \mathrm{mmol} / \mathrm{l}$ and/or 1 -h blood glucose (BG) $\geq 10 \mathrm{mmol} / \mathrm{l}$ and/or $2-\mathrm{h} \mathrm{BG} \geq 8.5 \mathrm{mmol} / \mathrm{l}$; (2) NGT pregnancies were identified when the 75-g OGTT FBG $<5.1 \mathrm{mmol} / \mathrm{l}$ and 1-h BG $<10 \mathrm{mmol} / \mathrm{l}$ and 2 -h $\mathrm{BG}<8.5 \mathrm{mmol} / \mathrm{l}$; (3) for women who had multiple pregnancies during the period of data collection, only the first pregnancy was included. Exclusion criteria were as follows: (1) pregnant women with liver or renal insufficiency, alanine aminotransferase or aspartate aminotransferase $>$ three times the upper limit of normal, glomerular filtration rate $<60 \mathrm{ml} \cdot \mathrm{min}^{-1} \cdot 1.73 \mathrm{~m}^{-2}$; (2) pregnant women with congenital heart disease or abnormal uterine development (e.g., unicornuate uterus, naive uterus, arcuate uterus, mediastinal uterus); (3) mental health disorders (e.g., schizophrenia, depression, bipolar disorder); (4) pregnant women with incomplete case data records.

\section{Collection of Clinical Data and Biochemical Measurements}

Maternal characteristics were obtained from early pregnancy cards and electronic medical records from our hospital, including age, height, pre-pregnancy body mass index (BMI), educational background, family history of diabetes, menstrual history, history of cesarean delivery, GDM, polycystic ovary syndrome, hypertension, fasting blood glucose (FBG) during the first trimester, and FBG/triglyceride (TG) during 14-20 gestational weeks.

\section{Covariates}

With consideration of relevant literature[18] and guidelines, age of delivery was divided into $19-29, \quad 30-34,35-39,40-44$, and $\geq 45$ years; BMI before pregnancy was divided into $<18.5$, 
$18.5-23.9,24.0-27.9$, and $\geq 28.0 \mathrm{~kg} / \mathrm{m}^{2}$; educational background was divided into bachelor degree and above, junior college, technical secondary school/high school, and junior high school and below; height was divided into $\leq 158, \quad 159-161, \quad 162-165, \quad$ and $\geq 166 \mathrm{~cm}$ according to the quartile; FBG in the first trimester was divided into $\leq 4.09,4.10-4.37$, $4.38-4.59,4.60-4.79,4.80-5.09$, and 5.10$7.00 \mathrm{mmol} / \mathrm{l}$; FBG at 14-20 gestational weeks was divided into $\leq 4.18,4.19-4.48,4.49-4.85$, and $4.86-7.00 \mathrm{mmol} / \mathrm{l}$; TG at $14-20$ gestational weeks was divided into $\leq 1.53,1.54-1.90$, $1.91-2.49$, and $\geq 2.50 \mathrm{mmol} / 1$.

\section{Statistical Analyses}

Statistical evaluation was performed by SPSS 23.0. Variables of non-normal distribution were presented as median (interquartile range [IQR]), and normally distributed variables were shown as mean $\pm \mathrm{SD}$. Continuous variables were compared with the Mann-Whitney $U$ test or Student's $t$ test. The $\chi^{2}$ and Fisher's exact tests were used for the analysis of categorical variables. A $p$ value $<0.05$ was considered statistically significant.

Logistic regression was used to explore the risk factors of GDM. Factors with $p<0.20$ were subjected to multi-factor logistic regression to establish an early prediction model of GDM. Factors with $p<0.05$ or odds ratio $(\mathrm{OR})>1.5$ were included as predictors in the GDM risk score table. The regression coefficient ( $\beta$ value) of each factor was multiplied by 10 and rounded to the nearest whole number to set up the risk score for the first trimester and risk score during 14-20 gestational weeks. The AUC of the receiver-operating characteristic (ROC) curve was used to evaluate the discriminative ability of the model. A Hosmer-Lemeshow (HL) test was used to reflect the model prediction degree of closeness between the predicted prevalence rate and the observed prevalence rate to evaluate the calibration ability of the prediction model.

\section{RESULTS}

\section{Clinical Characteristics of Women with NGT and GDM Between the Training Cohort and Internal Validation Cohort}

In total, 785 pregnant women with NGT and 855 pregnant women with GDM were included. Table 1 presents descriptive statistics at baseline of NGT and GDM pregnancies. In the training and internal validation cohorts, there were no statistically significant differences in menarche age, gravidity or parity. However, the maternal age, pre-pregnancy weight, and pre-pregnancy BMI were significantly higher in GDM pregnancies than in NGT pregnancies $(p<0.05$; Table 1).

Table 1 Clinical characteristics of women with NGT and GDM between training cohort and internal validation cohort

\begin{tabular}{|c|c|c|c|c|c|c|}
\hline & \multicolumn{3}{|c|}{ Training cohort } & \multicolumn{3}{|c|}{ Internal validation cohort } \\
\hline & $\begin{array}{l}\text { NGT } \\
n=539\end{array}$ & $\begin{array}{l}\text { GDM } \\
n=611\end{array}$ & $p$ & $\begin{array}{l}\text { NGT } \\
n=246\end{array}$ & $\begin{array}{l}\text { GDM } \\
n=244\end{array}$ & $p$ \\
\hline Maternal age (years) & $30.2 \pm 4.1$ & $31.0 \pm 4.4$ & $<0.01$ & $30.7 \pm 4.9$ & $30.7 \pm 6.0$ & 0.025 \\
\hline Pre-pregnancy weight $(\mathrm{kg})$ & $57.6 \pm 7.6$ & $60.9 \pm 9.1$ & $<0.01$ & $57.5 \pm 7.8$ & $59.7 \pm 9.2$ & 0.026 \\
\hline Maternal height $(\mathrm{cm})$ & $162.3 \pm 4.7$ & $161.6 \pm 4.3$ & $<0.01$ & $162.3 \pm 4.6$ & $161.0 \pm 4.9$ & $<0.01$ \\
\hline Pre-pregnancy BMI $\left(\mathrm{kg} / \mathrm{m}^{2}\right)$ & $21.5 \pm 3.1$ & $23.0 \pm 3.5$ & $<0.01$ & $21.7 \pm 3.2$ & $23.1 \pm 3.3$ & $<0.01$ \\
\hline Menarche age (years) & $15.0 \pm 3.0$ & $15.0 \pm 3.1$ & 0.850 & $15.0 \pm 3.4$ & $15.7 \pm 3.6$ & 0.088 \\
\hline Gravidity $\geq 4(\%)$ & $9(1.7)$ & $19(3.1)$ & 0.280 & $122(22.7)$ & $154(24.1)$ & 0.308 \\
\hline Parity $\geq 4(\%)$ & $103(19.1)$ & $149(24.4)$ & 0.107 & $62(25.2)$ & $49(20.1)$ & 0.281 \\
\hline
\end{tabular}

$B M I$ body mass index 
Table 2 Clinical and laboratory risk factors for GDM

\begin{tabular}{|c|c|c|c|}
\hline & $\boldsymbol{B}$ & $p$ & OR $(95 \% \mathrm{CI})$ \\
\hline Maternal age (years) & 0.047 & $<0.01$ & $1.049(1.020-1.078)$ \\
\hline Maternal height $(\mathrm{cm})$ & -0.037 & $<0.01$ & $0.964(0.939-0.989)$ \\
\hline \multicolumn{4}{|l|}{ Pre-pregnancy BMI $\left(\mathrm{kg} / \mathrm{m}^{2}\right)$} \\
\hline$<18.5$ & -0.315 & 0.170 & $0.730(0.466-1.144)$ \\
\hline $18.5-23.9$ & - & - & 1.000 \\
\hline $24.0-27.9$ & 0.671 & $<0.01$ & $1.956(1.428-2.681)$ \\
\hline$\geq 28$ & 1.027 & $<0.01$ & $2.792(1.640-4.753)$ \\
\hline Gravidity & 0.161 & 0.022 & $1.174(1.024-1.347)$ \\
\hline Parity & 0.246 & 0.103 & $1.278(0.951-1.718)$ \\
\hline Miscarriage & 0.202 & $<0.01$ & $1.224(1.067-1.404)$ \\
\hline Menstrual disorders & 1.100 & $<0.01$ & $3.005(1.514-5.965)$ \\
\hline IVF & 0.518 & 0.028 & $1.678(1.059-2.661)$ \\
\hline History of cesarean delivery & 0.702 & $<0.01$ & $2.019(1.376-2.961)$ \\
\hline History of GDM & 1.266 & 0.198 & $3.545(0.395-31.817)$ \\
\hline History of hypertension & 1.367 & $<0.01$ & $3.924(1.709-9.009)$ \\
\hline History of PCOS & 0.979 & 0.200 & $2.663(0.535-13.249)$ \\
\hline Family history of diabetes & 1.594 & $<0.01$ & $4.925(2.841-8.537)$ \\
\hline Neonatal sex (male) & 0.164 & 0.165 & $1.179(0.935-1.487)$ \\
\hline FBG during first trimester & 1.040 & $<0.01$ & $2.829(2.210-3.622)$ \\
\hline Elevated aminotransferase & 0.463 & 0.026 & $1.588(1.056-2.388)$ \\
\hline \multicolumn{4}{|c|}{ Examination at $14-20$ gestational weeks } \\
\hline $\mathrm{FBG}(\mathrm{mmol} / \mathrm{l})$ & 1.177 & $<0.01$ & $3.244(2.424-4.342)$ \\
\hline GGT (U/l) & 0.033 & $<0.01$ & $1.034(1.013-1.055)$ \\
\hline TG (mmol/l) & 0.720 & $<0.01$ & $2.054(1.622-2.601)$ \\
\hline $\mathrm{HDL}(\mathrm{mmol} / \mathrm{l})$ & -1.227 & $<0.01$ & $0.293(0.197-0.437)$ \\
\hline FT3 (pmol/l) & 0.296 & 0.071 & $1.345(0.975-1.855)$ \\
\hline FT4 (pmol/l) & -0.174 & $<0.01$ & $0.840(0.773-0.914)$ \\
\hline
\end{tabular}

Elevated aminotransferase: alanine aminotransferase and/or aspartate aminotransferase $>40 \mathrm{U} / \mathrm{l}$ $I V F$ in vitro fertilization, $P C O S$ polycystic ovary syndrome, $F B G$ fasting blood glucose, $G G T \gamma$-glutamyl transpeptidase, $T G$ triglyceride, $H D L$ high-density lipoprotein, FT3 free triiodothyronine, FT4 thyroxine 
Table 3 GDM risk score during the first trimester

\begin{tabular}{|c|c|c|c|c|c|c|c|c|c|}
\hline & $\beta$ & OR $(95 \% \mathrm{CI})$ & $p$ & Score & & $\beta$ & OR (95\% CI) & $p$ & Score \\
\hline $\begin{array}{l}\text { Maternal } \\
\text { height } \\
(\mathrm{cm})\end{array}$ & & & & & $\begin{array}{l}\text { Educational } \\
\text { background }\end{array}$ & & & & \\
\hline$\geq 166$ & - & 1 & 0.032 & 0 & University or above & - & 1 & $<0.01$ & 0 \\
\hline $162-165$ & 0.185 & $\begin{array}{l}1.20 \\
\quad(0.83-1.75)\end{array}$ & 0.334 & 2 & Junior college & 0.654 & $\begin{array}{l}1.92 \\
\quad(1.37-2.70)\end{array}$ & $<0.01$ & 7 \\
\hline $159-161$ & 0.446 & $\begin{array}{l}1.56 \\
\quad(1.05-2.32)\end{array}$ & 0.028 & 4 & $\begin{array}{l}\text { Technical } \\
\text { secondary and } \\
\text { high school }\end{array}$ & 1.018 & $\begin{array}{l}2.77 \\
\quad(1.55-4.96)\end{array}$ & $<0.01$ & 10 \\
\hline$\leq 158$ & 0.538 & $\begin{array}{l}1.71 \\
\quad(1.14-2.58)\end{array}$ & 0.010 & 5 & $\begin{array}{l}\text { Junior secondary } \\
\text { and below }\end{array}$ & 1.375 & $\begin{array}{l}3.96 \\
\quad(1.50-10.46)\end{array}$ & $<0.01$ & 14 \\
\hline $\begin{array}{c}\text { Pre-pregnanc } \\
\left(\mathrm{kg} / \mathrm{m}^{2}\right)\end{array}$ & cy BMI & & & & $\begin{array}{l}\text { Family history of } \\
\text { diabetes }\end{array}$ & & & & \\
\hline$<18.5$ & -0.079 & $\begin{array}{l}0.92 \\
\quad(0.57-1.51)\end{array}$ & 0.753 & -1 & No & - & 1 & - & 0 \\
\hline $18.5-23.9$ & - & 1 & 0.028 & 0 & Yes & 1.534 & $\begin{array}{l}4.64 \\
\quad(2.54-8.46)\end{array}$ & $<0.01$ & 15 \\
\hline $24.0-27.9$ & 0.441 & $\begin{array}{l}1.55 \\
\quad(1.10-2.20)\end{array}$ & 0.013 & 4 & $\begin{array}{l}\text { Menstrual } \\
\text { disorders }\end{array}$ & & & & \\
\hline$\geq 28.0$ & 0.585 & $\begin{array}{l}1.79 \\
\quad(0.97-3.34)\end{array}$ & 0.064 & 6 & No & - & 1 & - & 0 \\
\hline $\begin{array}{c}\text { FBG during } \\
\text { trimester }\end{array}$ & first & & & & Yes & 0.930 & $\begin{array}{l}2.54 \\
\quad(1.19-5.40)\end{array}$ & 0.016 & 9 \\
\hline$\leq 4.09$ & - & 1 & $<0.01$ & 0 & $\begin{array}{l}\text { History of } \\
\text { hypertension }\end{array}$ & & & & \\
\hline $4.10-4.37$ & 0.055 & $\begin{array}{l}1.06 \\
(0.67-1.67)\end{array}$ & 0.815 & 1 & No & - & 1 & - & 0 \\
\hline $4.38-4.59$ & 0.493 & $\begin{array}{l}1.64 \\
\quad(1.03-2.61)\end{array}$ & 0.038 & 5 & Yes & 1.002 & $\begin{array}{l}2.72 \\
\quad(1.49-4.99)\end{array}$ & 0.001 & 10 \\
\hline $4.60-4.79$ & 0.750 & $\begin{array}{l}2.12 \\
\quad(1.34-3.35)\end{array}$ & $<0.01$ & 8 & $\begin{array}{l}\text { History of cesarean } \\
\text { delivery }\end{array}$ & & & & \\
\hline $4.80-5.09$ & 0.919 & $\begin{array}{l}2.51 \\
\quad(1.61-3.92)\end{array}$ & $<0.01$ & 9 & No & - & 1 & - & 0 \\
\hline $5.10-7.00$ & 1.641 & $\begin{array}{l}5.16 \\
\quad(3.21-8.29)\end{array}$ & $<0.01$ & 16 & Yes & 0.437 & $\begin{array}{l}1.55 \\
\quad(0.99-2.43)\end{array}$ & 0.058 & 4 \\
\hline
\end{tabular}


Table 3 continued

\begin{tabular}{|c|c|c|c|c|c|c|c|c|c|}
\hline & $\beta$ & OR $(95 \% \mathrm{CI})$ & $p$ & Score & & $\beta$ & OR $(95 \% \mathrm{CI})$ & $p$ & Score \\
\hline $\begin{array}{l}\text { Maternal } \\
\text { age } \\
\text { (year) }\end{array}$ & & & & & History of GDM & & & & \\
\hline $19-29$ & - & 1 & 0.186 & 0 & No & - & 1 & - & 0 \\
\hline $30-34$ & 0.336 & $\begin{array}{l}1.40 \\
\quad(1.04-1.89)\end{array}$ & 0.027 & 3 & Yes & 1.178 & $\begin{array}{l}3.25 \\
\quad(0.31-33.88)\end{array}$ & 0.325 & 12 \\
\hline $35-39$ & 0.221 & $\begin{array}{l}1.25 \\
\quad(0.85-1.84)\end{array}$ & 0.264 & 2 & History of PCOS & & & & \\
\hline $40-44$ & 0.573 & $\begin{array}{l}1.77 \\
\quad(0.70-4.51)\end{array}$ & 0.228 & 6 & No & - & 1 & - & 0 \\
\hline$\geq 45$ & 1.013 & $\begin{array}{l}2.76 \\
\quad(0.22-34.62)\end{array}$ & 0.433 & 10 & Yes & 0.931 & $\begin{array}{l}2.54 \\
\quad(0.43-15.11)\end{array}$ & 0.306 & 9 \\
\hline
\end{tabular}

$B M I$ body mass index, PCOS polycystic ovary syndrome, IVF-ET in vitro fertilization and embryo transfer

Table 4 GDM risk score during 14-20 gestational weeks

\begin{tabular}{lllll}
\hline \multicolumn{1}{c}{$\boldsymbol{B}$} & OR $(\mathbf{9 5 \%} \mathbf{C I})$ & $\boldsymbol{p}$ & Score \\
\hline $\begin{array}{l}\text { FBG during } \\
\quad \text { 14-20 gestational weeks }\end{array}$ 4.18 & - & 1 & $<0.01$ & 0 \\
$4.19-4.48$ & 0.063 & $1.07(0.60-1.89)$ & 0.828 & 1 \\
$4.49-4.85$ & 0.874 & $2.40(1.34-4.28)$ & $<0.01$ & 9 \\
$4.86-7.00$ & 2.208 & $9.09(4.52-18.30)$ & $<0.01$ & 22 \\
TG & & & & 0 \\
$\leq 1.53$ & - & 1 & 0.014 & 6 \\
$1.54-1.90$ & 0.563 & $1.76(0.98-3.16)$ & 0.060 & 8 \\
$1.91-2.49$ & 0.781 & $2.18(1.21-3.93)$ & 0.009 & 9 \\
$\geq 2.50$ & 0.948 & $2.58(1.41-4.73)$ & 0.002 & \\
\hline
\end{tabular}

\section{Development of GDM Risk Score Prediction Model}

Clinical and Laboratory Risk Factors for GDM Explored by Univariable Logistic Regression Regarding clinical risk factors, maternal age and pre-pregnancy BMI were significantly and positively correlated with GDM risk. However, maternal height was significantly and inversely related to GDM risk (Table 2). Histories of in vitro fertilization (IVF), menstrual disorders, multiple pregnancies, miscarriage, cesarean delivery, and hypertension were also associated with a significantly increased risk of GDM. Women with a history of menstrual disorders, cesarean delivery, and hypertension were more likely to develop GDM (3.005-, 2.019-, and 3.924-fold, respectively). Additionally, a family 


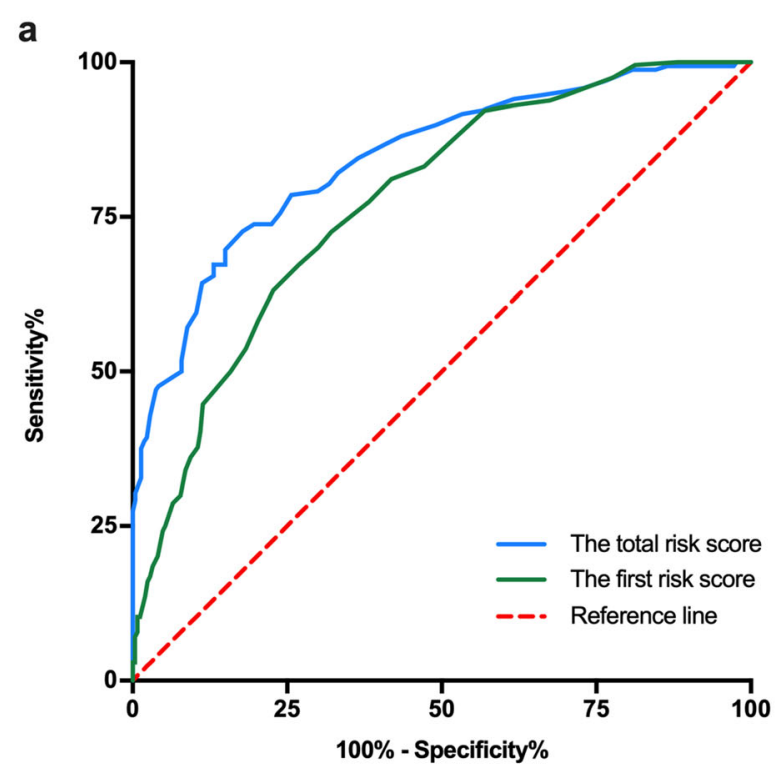

Fig. 1 Area under the receiver-operating characteristic curve (AUC) of the first risk score and the total risk score in the validation cohorts. a In the internal validation cohort, AUCs of the first risk scores and the total risk score predicting GDM were $0.774 \quad(95 \%$ $\mathrm{CI}=0.733-0.814)$ and $0.845(95 \% \mathrm{CI}=0.805-0.884)$,

history of diabetes significantly increased the risk of GDM, with an OR of 4.925, [95\% $\mathrm{CI}=2.841-8.537]$. Regarding laboratory risk factors, FBG, alanine aminotransferase (ALT), or aspartate aminotransferase (AST) during the first trimester as well as FBG, $\gamma$-glutamyl transpeptidase (GGT), and TG during 14-20 gestational weeks were significantly and positively correlated with GDM risk. However, high-density lipoprotein (HDL) and free thyroxine (FT4) during 14-20 gestational weeks were significantly and inversely related to GDM risk.

\section{Clinical and Laboratory Risk Factors for GDM Explored by Multivariate Logistic Regression}

In the multivariate logistic regression analysis, we developed the first risk score for the first trimester and the risk score for 14-20 gestational weeks. As shown in Table 3, maternal age, pre-pregnancy BMI, and FBG during the first trimester as well as educational background, menstrual disorders, and hypertension were independent predictors for GDM. Additionally, women with a history of cesarean delivery, b

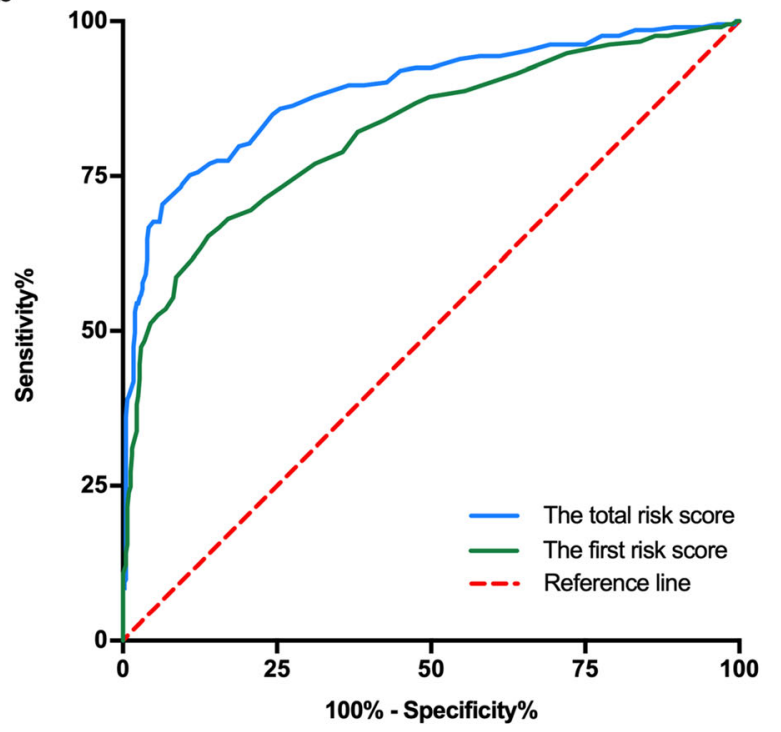

respectively. $\mathbf{b}$ In the external validation cohort, AUCs of the risk scores were $0.822(95 \% \mathrm{CI}=0.785-0.858)$ and $0.886(95 \% \mathrm{CI}=0.856-0.916)$, respectively

previous GDM, or polycystic ovarian syndrome (PCOS) also had a higher risk of developing GDM (all OR > 1.5).

A multivariate logistic analysis of clinical and laboratory risk factors indicated that FBG and TG during 14-20 gestational weeks were independent predictors for GDM (all $p<0.05$ ). Therefore, we developed a risk score for 14-20 gestational weeks using the same methods described above (Table 4).

\section{Risk Score Validation}

The final risk prediction models were tested within internal and external cohorts to assess both internal and external validity. In terms of discrimination, the first risk score tested within the internal cohort achieved an AUC of 0.774 (95\% CI $=0.733-0.814)$. After further including FBG and TG during 14-20 gestational weeks, discrimination of the total risk score improved to 0.845 (95\% CI = 0.805-0.884; Fig. 1a). In the external cohort, the first risk score achieved an AUC of $0.822(95 \% \mathrm{CI}=0.785-0.858)$. With the 

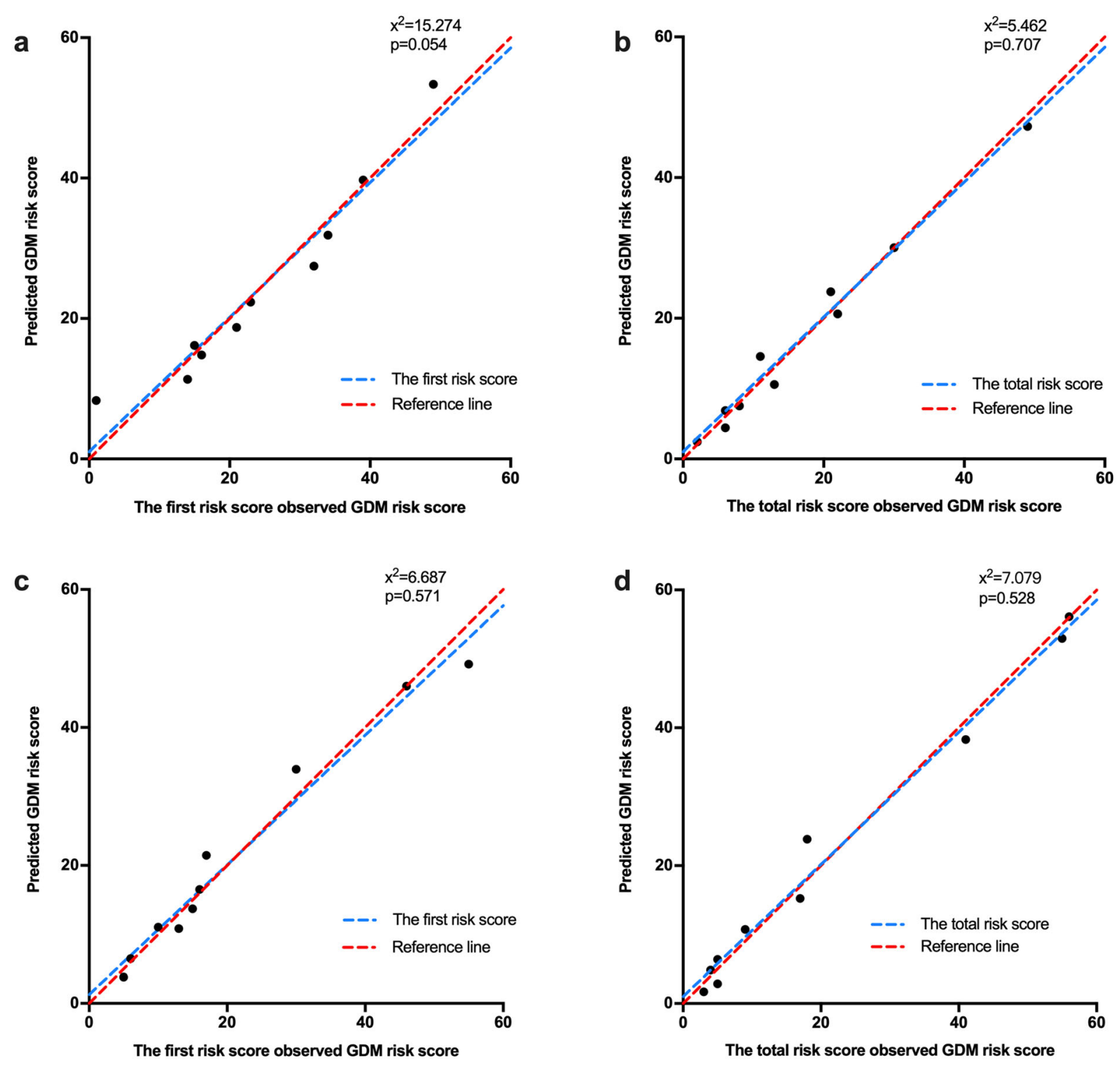

Fig. 2 Calibration curve for observed versus predicted risk of developing GDM in the internal cohort (a, b) and the external cohort $(\mathbf{c}, \mathbf{d})$

addition of FBG and TG during 14-20 gestational weeks, the AUC improved to 0.886 (95\% $\mathrm{CI}=0.856-0.916$; Fig. 1b).

Next, we estimated the sensitivity and specificity of the two risk scores. As for the first score tested in the internal cohort, at the cut-off point of 16.5 (total score $0-110$ ), the sensitivity was $72.5 \%$ and specificity was $67.9 \%$. After further including FBG and TG, and with a cut-off point of 28.5 (total score $0-141$ ), the sensitivity and specificity were $79.2 \%$ and $70.1 \%$, respectively. In the external cohort, at the cutoff point of 19.5 , the sensitivity and specificity for the first risk score were $77.0 \%$ and $68.8 \%$, respectively. After further including FBG and TG, at a cut-off point of 29.5, the sensitivity and specificity were $86.4 \%$ and $72.5 \%$, respectively.

Finally, the calibration of the two risk scores was evaluated by a Hosmer-Lemeshow goodness-of-fit test. In the internal cohort, the test of the first score indicated a good fit $\left(\chi^{2}=15.274\right.$; $p=0.054)$. After further including FBG and TG, 
the results still demonstrated an acceptable calibration $\left(\chi^{2}=5.462 ; p=0.707\right.$; Fig. $\left.2 \mathrm{a}, \mathrm{b}\right)$. In the external cohort, the Hosmer-Lemeshow test indicated acceptable calibration of these two risk scores $\left(\chi^{2}=6.687, p=0.571 ; \chi^{2}=7.079\right.$, $p=0.528$, respectively; Fig. 2 c, d). In the calibration curve, the dots were randomly distributed around $y=x$, indicating that the actual values were close to the predicted values (Fig. 2).

\section{DISCUSSION}

In this study, using a cohort of 1150 pregnant Chinese women (training cohort), we developed a simple, non-invasive, practical tool to predict undiagnosed GDM based on clinical characteristics and laboratory tests. We found that 11 predictors collected at the first antenatal care visit (i.e., maternal age, height, pre-pregnancy BMI, educational background, FBG during the first trimester, family history of diabetes, menstrual disorders, history of hypertension, cesarean delivery, GDM, and PCOS) and two laboratory risk factors (FBG and TG during 14-20 gestational weeks) were associated with an increasing risk of GDM. Most importantly, our risk scores demonstrated acceptable calibration (both $p$ for Hosmer-Lemeshow test $>0.05$ ) and discrimination in the external cohort, with the AUC for the first and the total risk score being $0.822(95 \% \mathrm{CI}=0.785-0.858)$ and 0.886 (95\% CI $=0.856-0.916)$, respectively. Collectively, our observations should encourage others to test and validate the simple GDM prediction models in different populations and to better identify women at high risk of developing GDM early in pregnancy.

Based on current guidelines, GDM is typically diagnosed using a 75-g oral glucose tolerance test conducted between 24 and 28 weeks of pregnancy $[11,12]$. However, previous studies suggested that fetal growth can already be abnormal preceding the diagnosis of GDM, including smaller fetuses at 24 weeks of gestation [19] and increased abdominal circumference growth rates compared with the non-GDM group [20]. Most studies showed that women with early GDM (before 24 weeks of gestation) are at high risk of adverse pregnancy outcomes
$[21,22]$, such as pregnancy-induced hypertension, hyperbilirubinemia, preterm birth, and perinatal mortality [23]. A recent Thailand study showed that early GDM women had higher rates of preeclampsia, LGA infants, and NICU admission [24]. Therefore, a hysteretic diagnosis of GDM might be too late for intervention and cannot completely reverse changes in epigenetics and abnormal fetal growth that occurred before 24 weeks of gestation. In this study, we established the GDM risk score to identify women at high risk of GDM before 24 weeks of gestation, allowing for early interventions and improved outcomes at birth.

Several studies confirmed that screening and treatment of gestational diabetes in high-risk women improves maternal and neonatal outcomes [25]. Early screening significantly reduced the rates of adverse outcomes in women compared to routine screening, such as emergency caesarean section, neonatal hypoglycemia, and macrosomia [26]. Furthermore, a recent study suggested that the immediate care of women with FBG levels $\geq 5.5 \mathrm{mmol} / \mathrm{l}$ may reduce rates of LGA infants, suggesting that such treatment should be immediately initiated in such cases [27]. Although several prediction models have been established to identify women at high risk of GDM before the diagnosis, such as the machine learning model [28] and multivariate regression model [14], they are not widely used in routine clinical practice. For this purpose, we generated a practical risk score consisting of routinely measured predictors, which performed well in an external cohort with the AUC of $0.886(95 \% \mathrm{CI}=0.856-0.916)$. Future prospective studies and studies on additional populations are needed to assess the realworld clinical utility of the risk score.

In recent years, several attempts have been made to combine clinical information and laboratory risk factors to screen women who are early in their pregnancy for the risk of developing GDM. Most previously reported GDM prediction models are from European and American populations [14, 15, 17, 29-34], with only a few from Asian and African populations [28, 35-37]. The laboratory risk factors in these models include FBG, glycosylated hemoglobin, the homeostasis model insulin resistance index, 
blood lipids, leptin, adiponectin, and lipocalin$2[17,34]$. However, except for FBG levels, most other indicators are not tested during routine antenatal care visits. Several studies confirmed that clinical factors, such as maternal age, prepregnancy BMI, gravidity, race, family history of diabetes, history of chronic hypertension, GDM, PCOS, diet, and exercise during pregnancy, are all related to GDM risk [17, 34, 36, 38]. Additionally, evidence showed that the waist-height ratio was the best predictor of glucose intolerance, followed by waist circumference, waist-hip ratio, and BMI, suggesting that height and waist circumference can affect glucose metabolism [39]. In this context, we also found that height was negatively correlated with the risk of GDM. However, data on the waist circumference of pregnant women are lacking. Whether the effect of height on glucose metabolism is independent of waist circumference needs further validation. Besides, we found that the lower the education level, the higher the proportion of pregnant women with GDM, which may be related to their lower education, economic level/family status, decreased social support, and/or consumption of large amounts of calories during pregnancy.

Our study has several strengths. First, the variables of the GDM risk score were collected from the routine antenatal care system in community hospitals during the first trimester of gestation and 14-20 weeks of gestation, which does not increase the additional medical burden to pregnant women. Second, our GDM risk score has a high predictive ability. Moreover, it demonstrated good generalizability and applicability in the external validated cohort from Suzhou Hospital Affiliated to Nanjing Medical University. Third, the GDM risk score is a simple and economical assessment tool that can be disseminated through the media, the Internet, specialized hospitals, and primary care facilities. Furthermore, it can be easily understood and recognized by a large number of nonprofessional groups in a short time.

Our study also has some limitations. First, our GDM risk score was developed and validated predominantly in a Chinese population. Further validation studies are required to evaluate the generalizability and applicability of our score to other populations in different settings. In addition, our risk score lacked measures of waist circumference, glycosylated hemoglobin, leptin, adiponectin, and other risk factors. Further studies are needed to determine whether it is recommended to detect these indicators during early pregnancy to predict the risk of GDM.

\section{CONCLUSIONS}

In summary, we propose a simple risk score based on clinical characteristics and laboratory biomarkers to better identify women at high risk of developing GDM early in pregnancy to facilitate early interventions and potentially reduce adverse outcomes. Further validation is needed to evaluate the performance of risk scores in different populations. The impact of early preventive and intervention strategies following positive early screening for GDM using the risk score should also be explored.

\section{ACKNOWLEDGEMENTS}

The authors would like to thank all volunteers for their participation in this study.

Funding. This work and the journal's Rapid Service Fee were sponsored by the grants from the National Natural Science Foundation of China Grant Award (81770819), Jiangsu Provincial Key Medical Discipline (ZDXKB2016012), and the Six Talent Peaks Project of Jiangsu Province of China (YY-086).

Authorship. All named authors meet the International Committee of Medical Journal Editors (ICMJE) criteria for authorship for this article, take responsibility for the integrity of the work as a whole, and have given their approval for this version to be published.

Authors' Contributions. YMW and BY developed the study. YMW, ZJG and LC conducted the study, performed the analysis and wrote the manuscript. YMW, JH and WTZ were involved in data collection and statistical analysis. YB and DLZ supervised the study and 
critically reviewed the manuscript for important intellectual content. LC collected the medical records of the external validation cohort from Suzhou Hospital Affiliated to Nanjing Medical University, Suzhou, China. SMS was in charge of revising the manuscript. All authors contributed to the interpretation of data and revision of the manuscript and provided final approval of the version to be published. All authors have read and approved the final manuscript.

Disclosures. Yanmei Wang, Zhijuan Ge, Lei Chen, Jun Hu, Wenting Zhou, Shanmei Shen, Dalong Zhu, and Yan Bi declare that they have nothing to disclose.

Compliance with Ethics Guidelines. This study was approved by the Institutional Ethics Committee of Nanjing Drum Tower Hospital, The Affiliated Hospital of Nanjing University Medical School, Nanjing, China. Our study conforms to the guidelines of the Declaration of Helsinki and its later amendments. Informed consent was obtained from all patients for being included in the study. Date of approval: 2016-03-29. Reference number: AF/SC-08/02.0.

Data Availability. The datasets generated and analyzed during the current study are available from the corresponding author on reasonable request.

Open Access. This article is licensed under a Creative Commons Attribution-NonCommercial 4.0 International License, which permits any non-commercial use, sharing, adaptation, distribution and reproduction in any medium or format, as long as you give appropriate credit to the original author(s) and the source, provide a link to the Creative Commons licence, and indicate if changes were made. The images or other third party material in this article are included in the article's Creative Commons licence, unless indicated otherwise in a credit line to the material. If material is not included in the article's Creative Commons licence and your intended use is not permitted by statutory regulation or exceeds the permitted use, you will need to obtain permission directly from the copyright holder. To view a copy of this licence, visit http://creativecommons.org/licenses/by$\mathrm{nc} / 4.0 /$.

\section{REFERENCES}

1. Gortazar L, Flores-Le Roux JA, Benaiges D, et al. Trends in prevalence of gestational diabetes and perinatal outcomes in Catalonia, Spain, 2006 to 2015: the Diagestcat Study. Diabetes Metab Res Rev. 2019;35:e3151.

2. Ovesen PG, Jensen DM, Damm P, Rasmussen S, Kesmodel US. Maternal and neonatal outcomes in pregnancies complicated by gestational diabetes. A nation-wide study. J Matern Fetal Neonatal Med. 2015;28:1720-4.

3. Fadl HE, Ostlund IK, Magnuson AF, Hanson US. Maternal and neonatal outcomes and time trends of gestational diabetes mellitus in Sweden from 1991 to 2003. Diabet Med. 2010;27:436-41.

4. Group HSCR, Metzger BE, Lowe LP, et al. Hyperglycemia and adverse pregnancy outcomes. N Engl J Med. 2008;358:1991-2002.

5. Dabelea D, Hanson RL, Lindsay RS, et al. Intrauterine exposure to diabetes conveys risks for type 2 diabetes and obesity: a study of discordant sibships. Diabetes. 2000;49:2208-11.

6. Damm P, Houshmand-Oeregaard A, Kelstrup L, Lauenborg J, Mathiesen ER, Clausen TD. Gestational diabetes mellitus and long-term consequences for mother and offspring: a view from Denmark. Diabetologia. 2016;59:1396-9.

7. Guillemette L, Wicklow B, Sellers EAC, et al. Intrauterine exposure to diabetes and risk of cardiovascular disease in adolescence and early adulthood: a population-based birth cohort study. CMAJ. 2020;192:E1104-13.

8. Kim C, Newton KM, Knopp RH. Gestational diabetes and the incidence of type 2 diabetes: a systematic review. Diabetes Care. 2002;25:1862-8.

9. Koivusalo SB, Rono K, Klemetti MM, et al. Gestational diabetes mellitus can be prevented by lifestyle intervention: the Finnish gestational diabetes prevention study (RADIEL). A Randomized Controlled Trial. Diabetes Care. 2016;39:24-30 (Diabetes Care. 2017;40:1133).

10. Song C, Li J, Leng J, Ma RC, Yang X. Lifestyle intervention can reduce the risk of gestational 
diabetes: a meta-analysis of randomized controlled trials. Obes Rev. 2016;17:960-9.

11. American Diabetes A. 2. Classification and diagnosis of diabetes: standards of medical care in diabetes-2020. Diabetes Care. 2020;43:S14-31.

12. Weinert LS. International Association of Diabetes and Pregnancy Study Groups recommendations on the diagnosis and classification of hyperglycemia in pregnancy: comment to the International Association of Diabetes and Pregnancy Study Groups Consensus Panel. Diabetes Care. 2010;33:e97 (author reply e8).

13. Phaloprakarn C, Tangjitgamol S, Manusirivithaya S. A risk score for selective screening for gestational diabetes mellitus. Eur J Obstet Gynecol Reprod Biol. 2009;145:71-5.

14. Sweeting AN, Appelblom H, Ross GP, et al. First trimester prediction of gestational diabetes mellitus: a clinical model based on maternal demographic parameters. Diabetes Res Clin Pract. 2017; 127:44-50.

15. Savvidou M, Nelson SM, Makgoba M, Messow CM, Sattar N, Nicolaides K. First-trimester prediction of gestational diabetes mellitus: examining the potential of combining maternal characteristics and laboratory measures. Diabetes. 2010;59: 3017-22.

16. Sweeting AN, Wong J, Appelblom H, et al. A first trimester prediction model for gestational diabetes utilizing aneuploidy and pre-eclampsia screening markers. J Matern Fetal Neonatal Med. 2018;31: 2122-30.

17. Sweeting AN, Wong J, Appelblom H, et al. A novel early pregnancy risk prediction model for gestational diabetes mellitus. Fetal Diagn Ther. 2019;45: 76-84.

18. Zhou X, Qiao Q, Ji L, et al. Nonlaboratory-based risk assessment algorithm for undiagnosed type 2 diabetes developed on a nation-wide diabetes survey. Diabetes Care. 2013;36:3944-52.

19. Sletner L, Jenum AK, Yajnik CS, et al. Fetal growth trajectories in pregnancies of European and South Asian mothers with and without gestational diabetes, a population-based cohort study. PLOS ONE. 2017; 12:e0172946.

20. Sovio U, Murphy HR, Smith GC. Accelerated fetal growth prior to diagnosis of gestational diabetes mellitus: a prospective cohort study of Nulliparous women. Diabetes Care. 2016;39:982-7.

21. Liu B, Cai J, Xu Y, et al. Early diagnosed gestational diabetes mellitus is associated with adverse pregnancy outcomes: a prospective cohort study. J Clin Endocrinol Metab. 2020;105:e4264-74.

22. Sweeting AN, Ross GP, Hyett J, et al. Gestational diabetes mellitus in early pregnancy: evidence for poor pregnancy outcomes despite treatment. Diabetes Care. 2016;39:75-81.

23. Barahona MJ, Sucunza N, Garcia-Patterson A, et al. Period of gestational diabetes mellitus diagnosis and maternal and fetal morbidity. Acta Obstet Gynecol Scand. 2005;84:622-7.

24. Boriboonhirunsarn D, Sunsaneevithayakul P, Pannin C, Wamuk T. Prevalence of early-onset GDM and associated risk factors in a university hospital in Thailand. J Obstet Gynaecol. 2020. https://doi.org/ 10.1080/01443615.2020.1820469.

25. De Muylder X. Perinatal complications of gestational diabetes: the influence of the timing of the diagnosis. Eur J Obstet Gynecol Reprod Biol. 1984;18:35-42.

26. Ryan DK, Haddow L, Ramaesh A, et al. Early screening and treatment of gestational diabetes in high-risk women improves maternal and neonatal outcomes: a retrospective clinical audit. Diabetes Res Clin Pract. 2018;144:294-301.

27. Cosson E, Vicaut E, Berkane N, et al. Prognosis associated with initial care of increased fasting glucose in early pregnancy: a retrospective study. Diabetes Metab. 2020. https://doi.org/10.1016/j. diabet.2020.08.007.

28. Wu YT, Zhang CJ, Mol BW, et al. Early prediction of gestational diabetes mellitus in the Chinese population via advanced machine learning. J Clin Endocrinol Metab. 2020;106:e1191-205.

29. Artzi NS, Shilo S, Hadar E, et al. Prediction of gestational diabetes based on nationwide electronic health records. Nat Med. 2020;26:71-6.

30. Badon SE, Zhu Y, Sridhar SB, et al. A pre-pregnancy biomarker risk score improves prediction of future gestational diabetes. J Endocr Soc. 2018;2:1158-69.

31. Savona-Ventura C, Vassallo J, Marre M, Karamanos BG, group M-Gs. A composite risk assessment model to screen for gestational diabetes mellitus among Mediterranean women. Int J Gynaecol Obstet. 2013;120:240-4.

32. Sridhar SB, Xu F, Darbinian J, Quesenberry CP, Ferrara A, Hedderson MM. Pregravid liver enzyme levels and risk of gestational diabetes mellitus during a subsequent pregnancy. Diabetes Care. 2014;37:1878-84. 
33. Theriault S, Forest JC, Masse J, Giguere Y. Validation of early risk-prediction models for gestational diabetes based on clinical characteristics. Diabetes Res Clin Pract. 2014;103:419-25.

34. Theriault S, Giguere Y, Masse J, Girouard J, Forest JC. Early prediction of gestational diabetes: a practical model combining clinical and biochemical markers. Clin Chem Lab Med. 2016;54:509-18.

35. Kouhkan A, Khamseh ME, Moini A, et al. Diagnostic accuracy of body mass index and fasting glucose for the prediction of gestational diabetes mellitus after assisted reproductive technology. Int J Fertil Steril. 2019;13:32-7.

36. Nombo AP, Mwanri AW, Brouwer-Brolsma EM, Ramaiya KL, Feskens EJM. Gestational diabetes mellitus risk score: a practical tool to predict gestational diabetes mellitus risk in Tanzania. Diabetes Res Clin Pract. 2018;145:130-7.

37. Zhang $\mathrm{X}$, Zhao $\mathrm{X}$, Huo L, et al. Risk prediction model of gestational diabetes mellitus based on nomogram in a Chinese population cohort study. Sci Rep. 2020;10:21223.

38. Laine MK, Kautiainen H, Gissler M, et al. Short primiparous women are at an increased risk for gestational diabetes mellitus. Public Health. 2018;156:101-8.

39. Lawal Y, Bello F, Anumah FE, Bakari AG. Waistheight ratio: How well does it predict glucose intolerance and systemic hypertension? Diabetes Res Clin Pract. 2019;158:107925. 